\title{
Recommendations for use of the polymerase chain reaction in the diagnosis of Bordetella pertussis infections*
}

\author{
B. D. MEADE and A. BOLLEN $\dagger$
}

Division of Bacterial Products, HFM-490, Center for Biologics Evaluation and Research, Food and Drug Administration, 1401 Rockville Pike, Rockville, MD 20852-1448, USA and $\dagger$ Applied Genetics, Université Libre de Bruxelles, Rue de l'Industrie 24, B-1400 Nivelles, Belgium

\section{Introduction}

Epidemiological surveys of Bordetella pertussis infections, as well as studies on the efficacy of ongoing pertussis vaccine trials, rely on cultures from nasopharyngeal specimens and on serology. ${ }^{1-4}$ While these two techniques together have markedly increased the detection capability of either alone, it remains clear that only a portion of clinically diagnosed cases can be confirmed. In the last few years, diagnostic systems based on the polymerase chain reaction (PCR) have been described. ${ }^{5-13}$ However, differences in the choice of target genes and amplification primers, as well as in the methodology itself, have not allowed a consensus to be reached on the usefulness of the PCR assay for the case definition of pertussis.

In May 1993, representatives from a majority of laboratories employing PCR for B. pertussis detection and identification were brought together in Rixensart (Belgium) under the sponsorship of the SmithKline Biologicals Company. The goals of the meeting were to exchange information, to reach a consensus on methodology where possible, to discuss technical issues and to make recommendations useful to investigators involved in efficacy trials of acellular pertussis vaccines.

\section{Discussion topics}

\section{Sample collection and processing}

Two types of samples were considered: those resulting from nasopharyngeal aspirates (NPA) and those collected with nasopharyngeal swabs (NPS). Two research groups had good success with a simple procedure in which the NPA was first treated with a mucolytic agent $(\mathrm{N}$-acetylcysteine or sodium hydrox-

Received 2 Dec. 1993 ; accepted 25 Jan. 1994.

* Report of a round-table meeting held in Rixensart, Belgium, on 12 May 1993. ide), then centrifuged to pellet the bacteria. After careful removal of the supernatant fluid, the pellet was resuspended in water and boiled. The procedure offered several advantages such as simplicity, concentration of the bacteria and removal of potentially PCR-inhibiting substances. In other laboratories, additional steps have been tested, such as the use of Chelex 100 to remove inhibitors or phenol-chloroform extraction of DNA before amplification. Long-term storage by freezing of NPA appeared suitable to preserve the sample for PCR, although no extensive validation was presented.

Several groups reported successful results with NPS as the source material, although there was little agreement on the optimal method of collection. Nevertheless, it appeared that Dacron swabs were preferred to calcium alginate, especially when transport medium was employed, as the latter tended to pull off the swab-stick or fall apart. Processing of dry NPS was less complicated and required fewer steps than processing of swabs stored in transport medium. For the latter specimens, DNA extraction appeared to be necessary, as there may be inhibitory factors in the transport medium. Dr Müller reported that DNA extraction from NPS with Chelex 100 provided a simple, rapid and effective method. Sufficient material for PCR could be obtained from dry NPS either by boiling in the presence of Chelex 100 or by elution and extraction of DNA. The use of frozen swabs had not been thoroughly investigated and, pending further studies, it would seem prudent to process the swab and freeze the material after centrifugation of bacteria or extraction of the DNA. For clinical trials in which swabs are to be used, some participants recommended that two swabs be collected, one for culture and the other for PCR. However, in Dr Müller's opinion, it might be better to use only a single swab for both assays because, in his experience, there has been frequent variability in the quality of consecutive swabs collected from the same patient.

To conclude this part of the discussion, participants 
agreed that sample processing should be as simple as possible to minimise the chances of loss of material or contamination. When possible, NPA would be preferable to swabs for PCR, primarily because of sensitivity and ease of processing. NPA also tended to be better than swabs for successful culture of $B$. pertussis. ${ }^{14}$ However, collecting NPA requires specialised equipment and appropriate training in order to collect proper samples. Most physicians and nurses generally have more experience with swabs and this may be the practical reason for using swabs in some trials.

\section{Primer selection and $P C R$ conditions}

Primers derived from four chromosomal regions have been used: (a) the pertussis toxin promoter region $;^{5,10,11,15}$ (b) a DNA region upstream of the porin gene (as described by $\mathrm{Dr} \mathrm{Li}$ ); (c) repeated insertion sequences; ${ }^{5-7,9,13}$ and (d) the adenylate cyclase toxin (ACT) gene. ${ }^{12}$

Studies examining the sensitivity and specificity were presented or have been published for all of these target loci. However, there were too few data to strongly advocate one in preference to the others. All seemed to be acceptable, except that the ACT primers did not distinguish between $B$. pertussis and $B$. parapertussis. There was some evidence that assays with the repeat sequence as target were more sensitive when a low number (20-25) of amplification cycles was used, but assays with all the targets were of comparable sensitivity if $>35$ cycles were used. A low number of amplification cycles reduced the incidence of false positive results, although the use of a system to prevent carry-over ${ }^{16}$ also seemed to minimise this problem. Primers to all four sequences were tested with DNA from a wide variety of bacteria, including strains of many species found in the respiratory tract. In no case was there a PCR-positive strain that was not of the genus Bordetella.

Some of the presentations described the use of nested primer systems and suggested that such systems offer advantages in sensitivity and specificity. In a nested system, about 20-25 cycles of amplification are performed with two outer primers followed by $25-30$ cycles of amplification with two inner primers. A disadvantage is that the uracil N-glycosylase (UNG) carry-over prevention system ${ }^{16}$ cannot be used before the second amplification.

One difference between the PCR systems described was whether or not they could distinguish $B$. pertussis from other species of Bordetella. One system produced a PCR product only with $B$. pertussis, another one with more than one species of Bordetella, and two of the systems could distinguish $B$. pertussis from $B$. parapertussis and $B$. bronchiseptica. For vaccine efficacy trials, it was agreed that the primer system selected should be able to detect both $B$. pertussis and $B$. parapertussis at the same time and distinguish between them, e.g., as in the systems described by Van der Zee et $a l^{13}$ and Reizenstein et al. ${ }^{15}$ If this proves too complex to implement, separate primer systems should be set up and used in parallel.

\section{Detection systems}

Although most detection systems employed ethidium bromide staining of bands in agarose gels, several others were discussed. These included the digoxigenin immunoblot system and either Southern blotting or dot blotting with radiolabelled probes. Again, because the systems have not been directly compared, it was not possible to determine which was best. However, desirable characteristics for a system for use in a clinical trial were discussed. Specifically, the use of an automated, quantitative system was preferred over subjective, visual reading of bands. Although several automated systems were described, none had been fully evaluated and a high priority should be given to their development and validation. In the absence of automated reading, blinded reading by laboratory staff was considered to be essential, and each test run should include coded positive and negative samples. Furthermore, laboratories should confirm results that are questionable. Proposals for such confirmation included digesting the amplified product into restriction fragments of defined size, demonstrating that the product binds specific labelled probes, or repeating the test with a different primer system either in the same or a different laboratory.

\section{Controls}

False positive reactions. The following were identified as possible causes of a false positive reaction, i.e., a positive PCR result with a sample from a patient not infected with $B$. pertussis: the sample contained other bacteria with sequences homologous to those in $B$. pertussis; contamination of sample with DNA or bacteria from laboratory strains of $B$. pertussis or another Bordetella sp.; product carry-over (contamination of samples with DNA from previous amplifications).

For clinical trials, the problem with false positive results was considered to be critical, and must be addressed thoroughly before employing PCR in case definition. Several steps have been used to minimise this problem, including physical separation of sample processing areas from amplification and detection areas and incorporation of both known negative samples and coded ("blinded") negative samples.

Product carry-over can be minimised by the use of the carry-over prevention system that destroys the product from previous amplifications. The most commonly used system is the uracil N-glycosylase (UNG) system $^{16}$ and its use was highly recommended where possible.

False negative results. The following were identified as possible causes of a false negative result, i.e., a negative PCR result with a sample collected from an individual infected with $B$. pertussis: too few bacteria in the nasopharynx; too few bacteria in the sample 
because of inadequate sampling procedure; presence of an inhibitor in the sample; bacteria or DNA lost or damaged during processing or storage; technical problems with assay (buffers, primers, instrumentation, etc.) low sensitivity of the detection system; patient infected with a $B$. pertussis strain with an altered or mutated sequence in the region defined by the primers.

Although it is not possible to control rigorously for all these, two groups described the use of internal controls for dealing with the problem of false negative results. In one system, ${ }^{5}$ each processed sample was amplified in two different PCR systems; one with $B$. pertussis primers and the other with primers for human DNA. As all NPAs are likely to contain some human cells, the sample should give a positive result for human DNA if it is collected, processed and amplified correctly.

Ms Houard proposed the following algorithm for evaluating the results of such a system:

Human positive, $B$. pertussis positive $=$ Infected with B. pertussis.

Human positive, $B$. pertussis negative $=$ Not likely to be infected with $B$. pertussis.

Human negative, $B$. pertussis negative $=$ Indeterminate, repeat amplification on same sample; try to collect and test a new sample.

Human negative, $B$. pertussis positive $=$ Indeterminate, repeat amplification on same sample; try to collect and test a new sample.

Dr Van der Zee ${ }^{13}$ proposed another internal control system in which a hybrid piece of DNA was added to each sample. In this system, primers Bp1 and Bp2 were specific for $B$. pertussis, and $\mathrm{BppX}$ and $\mathrm{BppZ}$ were specific for $B$. parapertussis. Into each PCR tube was added DNA from a hybrid plasmid containing regions homologous to Bpl and BppZ. Thus, when amplification had proceeded satisfactorily, a product of a unique size representing the $\mathrm{Bp} 1-\mathrm{BppZ}$ product would be seen.

The use of some type of internal control system was thus strongly recommended to control for false negative results.

\section{Validation of the diagnostic system}

To validate the diagnostic system, the participants agreed that the following points should be considered:

1. Sensitivity: (a) demonstration that nearly $100 \%$ of culture-positive samples are PCR positive; (b) demonstration that a variety of $B$. pertussis strains all react, including laboratory strains and clinical isolates obtained at different times and from different geographic areas; (c) positive DNA control given as the lowest possible detectable level of DNA (about five bacteria per PCR assay).

2. Specificity: demonstration that no reaction is observed when various non-Bordetella species are tested, including strains from species that would normally be isolated from the respiratory tract.
3. Inclusion of an internal control that would test for the quality of the amplification and detection systems.

4. Rigorous procedures to avoid, test and control for false positive results, including: (a) use of the UNG carry-over prevention system; (b) evaluation of a large number of known negative samples to estimate the rate of false positive results; (c) automated or blinded reading of PCR results; (d) inclusion in all assays of coded control samples (known positive and known negative samples); (e) confirmation of questionable results by a second method.

\section{Use of PCR in clinical trials}

Dr Li offered the following summary of six points that favoured the use of PCR in clinical trials of pertussis vaccines:

1. All available data indicated that the various PCR procedures had a diagnostic sensitivity at least comparable to, and in many cases more sensitive than, culture.

2. All available data indicated that the PCR methods presented were specific. Although some systems did not distinguish $B$. pertussis from other Bordetella $\mathrm{spp}$, no-one reported a positive reaction with a species not in the genus Bordetella.

3. PCR offers a greater possibility than culture or serology of detecting infection in asymptomatic, mildly symptomatic, or previously immunised individuals, and thus will assist in defining more clearly the epidemiology of pertussis.

4. PCR is more likely to detect infection in individuals treated with antibiotics, because even antibioticdamaged bacteria would be detected by PCR at a time when they may not be detected by culture.

5. Several pertussis vaccine efficacy trials are in progress and it is unlikely that further trials will be conducted. Therefore, it is essential that diagnostic procedures used in the current trials provide the most accurate and complete information available.

6. The information provided by PCR may justify the additional cost.

Overall, there appeared to be a consensus that PCR was ready to be applied to field studies, provided that it was applied cautiously and that the systems used were thoroughly validated and well controlled.

\section{Conclusions and recommendations}

PCR appears to be a rapid, sensitive, and specific technique that is likely to have an important role in pertussis diagnosis. Although each of the methods discussed appeared to be suitable, none had been directly compared to any other. Not only did the PCR methods differ, but also the study populations, methods of collection and preparation of clinical samples, and culture techniques. The studies differed 
markedly in the frequency with which culture-positive samples were observed. Therefore, there was no reference point that allowed a comparison of the PCR procedures. For example, all laboratories were able to score $80-100 \%$ of the culture-positive samples as PCR-positive but there were large differences in the percentage of PCR-positive samples that were culturenegative. This varied between 13 and $88 \%$ in the different studies. The difference could have been due to the sensitivity and specificity of the PCR methods, but was more likely to be the result of differences in study population (vaccination status, antibiotic usage, etc.), clinical samples (type, source, stages of disease), and culture procedures.

The ongoing field trials of acellular pertussis vaccine in various parts of the world offer an excellent opportunity to properly evaluate the use of PCR in pertussis diagnosis, and investigators were encouraged to incorporate PCR methods in these trials where possible, at least for the purpose of evaluating and validating the methodology.

The group of experts gave a cautious recommendation to investigators involved in ongoing trials that PCR could be included in the case definition of pertussis provided that the following conditions are met:

(a) The laboratory adopts a programme for the rigorous control of false-positive and false-negative results. This should include physical separation of sample processing and product detection.

(b) Before employing the method, the laboratory evaluates a reasonable number $(>100)$ of known negative samples to estimate the diagnostic specificity. These samples should be collected from normal healthy individuals as well as from individuals with respiratory diseases that have been confirmed to be caused by agents other than $B$. pertussis.

(c) All samples are labelled and coded so that laboratory personnel who perform the PCR analyses and read the results have no information about the patients or samples.

(d) All assays include coded control samples (known positive and negative samples) that are handled in such a way that their identity is not known by the laboratory personnel who process the samples and read the results.

\section{References}

1. Onorato IM, Wassilak SGF. Laboratory diagnosis of pertussis : the state of the art. Pediatr Infect Dis J 1987; 6: 145-151.

2. Lawrence AJ, Paton JC. Efficacy of enzyme-linked immunosorbent assay for rapid diagnosis of Bordetella pertussis infection. J Clin Microbiol 1987; 25: 2102-2104.

3. Long SS, Welkon CJ, Clark JL. Widespread silent transmission of pertussis in families : antibody correlates of infection and symptomatology. J Infect Dis $1990 ; 161: 400-486$.

4. Viljanen MK, Ruuskanen O, Granberg C, Salmi TT. Serological diagnosis of pertussis: IgM, IgA and IgG antibodies against Bordetella pertussis measured by enzyme-linked immunosorbent assay (ELISA). Scand J Infect Dis 1982; 14: $117-122$

5. Houard S, Hackel C, Herzog A, Bollen A. Specific identification (e) Any questionable results are confirmed by a secondary method (second amplification, binding with a specific probe, obtaining characteristic bands after restriction endonuclease cleavage, or repeating the test with a different method, either in the same of a different laboratory).

(f) PCR-positive results are accepted only in individuals with classical symptoms of pertussis. The clinical and epidemiological significance of a PCRpositive result in someone with mild or no symptoms should be interpreted with caution and, if possible, other markers such as serology or epidemiology should be added.

The most important conclusion of the meeting was that it is too early to recommend a standard PCR technique for detection of $B$. pertussis in clinical specimens, primarily because no comparative studies have been done. A strong recommendation of the group of experts was that a collaborative study should be planned to compare directly the various techniques available in order to formulate a standardised protocol widely applicable to all epidemiological surveys and vaccine trials.

Participants at the meeting were: H. Bogaerts, V. Melot and J. Pètre, SmithKline Biologicals, rue de l'Institut 89, 1330 Rixensart, Belgium; A. Bollen and S. Houard, Applied Genetics, Université Libre de Bruxelles, rue de l'Industrie 24, B-1400 Nivelles, Belgium; $\mathrm{X}$. Haerden, Connaught, 1755 Steeles Avenue West, Willowdale, Ontario, Canada M2R 3T4; J. Mertsola and Qiushui He, National Public Health Institute of Finland, Kiinamyllynkatu 13, Turku, SF20520, Finland; F. M. Müller, Children's Hospital, Johannes Gutenberg University, Laugenbeckstrasse 1, 55131 Mainz, Germany; W. von König, Institut für Hygiene, Städt, Krankenanst, Krefeld 4150, Germany; P. Mastrantonio and P. Stefanelli, Istituto Superiore di Sanità, Laboratorio di Batteriologia e Micologia Medica, viale Regina Elena 299, Roma 00161, Italy; G. Ratti, Biocine-Sclavo, I.R.I.S., via Fiorentina 1, 53100 Siena, Italy; A. Van der Zee, National Institute of Public Health and Environmental Protection, P.O. Box 1, 3720 BA, Bilthoven, The Netherlands; H. Hallander, L. Mardin and E. Reizenstein, Statens Bakteriologiska Laboratory, The National Bacteriology Laboratory, Lundagatan 2, 10521 Stockholm, Solna, Sweden; P. Olcén, Department of Clinical Microbiology and Immunology, Örebro Medical Center Hospital, S-70185 Örebro, Sweden; J. Storsaeter, Barnhälsovarden, Drakenbergsgatan 39, S-11741 Stockholm, Sweden; G. Schläpfer, University Children's Hospital, Department of Clinical Microbiology, Römergasse 8, CH-4000 Basel 5, Switzerland; J. Coote, Microbiology Department, Glasgow University, Glasgow G12 8QQ, United Kingdom; B. M. Meade and Z. M. Li, Division of Bacterial Products HSM-434, Center for Biologics Evaluation and Research, Food and Drug Administration, 1401 Rockville Pike, Rockville, MD 20852-14478, USA.

of Bordetella pertussis by the polymerase chain reaction. Res Microbiol 1989; 140: 477-487.

6. Glare EM, Paton JC, Premier RR, Lawrence AJ, Nisbet IT. Analysis of a repetitive DNA sequence from Bordetella pertussis and its application to the diagnosis of pertussis using the polymerase chain reaction. J Clin Microbiol 1990; 28: 1982-1987.

7. Olcén P, Bäckman A, Johansson B et al. Amplification of DNA by the polymerase chain reaction for the efficient diagnosis of pertussis. Scand J Infect Dis 1992; 24: 339-345.

8. Rossau R, Michielsen A, Jannes G, Duhamel M, Kersters K, van Heuverswijn $H$. DNA probes for Bordetella species and a colorimetric reverse hybridization assay for the detection of Bordetella pertussis. Mol Cell Probes 1992; 6: 281-289.

9. He Q, Mertsola J, Soini H, Skurnik M, Ruuskanen O, Viljanen 
MK. Comparison of polymerase chain reaction with culture and enzyme immunoassay for diagnosis of pertussis. J Clin Microbiol 1993; 31: 642-645.

10. Grimprel E, Bégué P, Anjak I, Betsou F, Guiso N. Comparison of polymerase chain reaction, culture, and Western immunoblot serology for diagnosis of Bordetella pertussis infection. J Clin Microbiol 1993; 31: 2745-2750.

11. Schläpfer G, Senn HP, Berger R, Just M. Use of the polymerase chain reaction to detect Bordetella pertussis in patients with mild or atypical symptoms of infection. Eur $J$ Clin Microbiol Infect Dis 1993; 12: 459-463.

12. Douglas E, Coote JG, Parton R, McPheat W. Identification of Bordetella pertussis in nasopharyngeal swabs by $\mathrm{PCR}$ amplification of a region of the adenylate cyclase gene. $J$ Med Microbiol 1993; 38: 140-144.

13. Van der Zee A, Agterberg C, Peeters M, Schellekens J, Mooi F.
Polymerase chain reaction assay for pertussis: simultaneous detection and discrimination of Bordetella pertussis and Bordetella parapertussis. J Clin Microbiol 1993; 31 : 2134-2140.

14. Hallander HO, Reizenstein E, Renemar B, Rasmuson G, Mardin L, Olin P. Comparison of nasopharyngeal aspirates with swabs for culture of Bordetella pertussis. J Clin Microbiol 1993; 31 : 50-52.

15. Reizenstein E, Johansson B, Mardin L, Abens J, Möllby R, Hallander HO. Diagnostic evaluation of PCR discriminative for Bordetella pertussis, parapertussis and bronchiseptica. Diagn Microbiol Infect Dis 1994; 17: 185-191.

16. Pang J, Modlin J, Yolken R. Use of modified nucleotides and uracil-DNA glycosylase (UNG) for the control of contamination in the PCR-based amplification of RNA. Mol Cell Probes 1992; 6: 251-256. 